\title{
Laboratório virtual de Física Moderna: sistema para espectrometria gama $^{+*}$
}

Nelson Canzian da Silva ${ }^{1}$

Departamento de Física - UFSC

Florianópolis - SC

\section{Resumo}

O artigo descreve como foi implementada uma simulação computacional de um sistema de detecção de radiação ionizante semelhante ao encontrado em tradicionais laboratórios de física moderna. A simulação modela um sistema composto por "fontes" radioativas emissoras de fótons com energias bem definidas e de um "detector" de comportamento semelhante a um sistema de espectrometria de fótons (cintilador + fotomultiplicadora + analisador multicanal). Como em um sistema real, além de escolher a fonte, é possível ajustar a tensão na fotomultiplicadora, o ganho do amplificador e o ganho de conversão do analisador multicanal, observando-se o efeito disso nos "dados" adquiridos. São apresentados e discutidos resultados obtidos com o simulador para alguns roteiros de experimentos (calibração em energia, identificação de energias desconhecidas, determinação da resolução em energia etc.). A simulação foi utilizada com duas turmas do curso de licenciatura em física a distância da UFSC em 2011 e em 2014, associadas à realização do experimento real. Permeando o texto são discutidas as motivações e os problemas do uso das simulações neste contexto. À guisa de conclusão é discutido como o trabalho se relaciona às principais metas dos laboratórios introdutórios de física, segundo um comitê da American Physical Society especificamente constituído para isso.

Palavras-chave: Laboratório de Física Moderna; Simulação; Detecção da radiação.

\footnotetext{
${ }^{+}$Modern physics virtual laboratory: gamma spectrometer system

* Recebido: setembro de 2014. Aceito: março de 2015.

${ }^{1}$ E-mail: nelson.canzian@ufsc.br
} 


\begin{abstract}
This paper describes how a computer simulation of an ionizing radiation detection system similar to those found in traditional modern physics laboratories was implemented. The simulation models a system composed of radioactive "sources" of photons with well-defined energies and a "detector" that works like a photon spectrometer (scintillator + photomultiplier tube + multichannel analyzer). In a real system, besides choosing the source, it is possible to adjust the high voltage applied at the photomultiplier tube, the gain of the analog amplifier and the gain of the conversion of the multichannel analyzer by observing the corresponding effects in the acquired "data". There are presented and discussed results obtained through the simulator for some experimental set-ups (energy calibration, identification of unknown energies, system energy resolution, etc.). The simulation was used with two groups of undergraduate students from a distance learning Physics course at UFSC in 2011 and 2014, along with the execution of the real experiment. Throughout the text, there are comments on the motivation and problems of using simulations in this context. Finally, the way this work relates to the main goals of introductory Physics laboratories is discussed, according to an American Physical Society committee especially created to evaluate that.
\end{abstract}

Keywords: Modern Physics Laboratory; Simulation; Radiation detection.

\title{
I. Introdução
}

As radiações ionizantes estão cada vez mais presentes no cotidiano. Além do interesse despertado por acidentes como o de Fukushima, são conhecidas também suas aplicações na medicina (radiologia convencional, tomografia computadorizada, medicina nuclear, radioterapia) e seu uso crescente e estratégico na indústria, como na esterilização de medicamentos (FAIRAND; FIDOPIASTIS, 2010; JACOBS, 1995; GUIDOLIN et al., 1988) e instrumentação cirúrgica (IPEN, 2011), inativação de sementes (MAITY et el., 2009), fiscalização aduaneira (GIBLIN; LIPTON, 2011; BRENNER, 2011; VALENCIA; MILLER, 2011) e caracterização de materiais (STOCK, 2008). Na ciência básica as radiações ionizantes estão presentes em praticamente todas as áreas: dos fundamentos da genética (WATSON; CRICK, 1953) à astronomia (GUDEL, 2009), passando pelos grandes laboratórios de física de partículas (ATLAS, 2008). 
Tendo em perspectiva essa ubiquidade das radiações ionizantes, parece-nos importante sensibilizar estudantes de vários perfis sobre o tema. Estudantes das ciências básicas e das áreas tecnológicas, das áreas médica e sanitarista e de cursos técnicos de enfermagem e radiologia têm interesse profissional, mas aprender mais sobre radiações e a estrutura da matéria poderia ser muito benéfico para a formação de um cidadão durante o ensino médio.

Atividades experimentais utilizando radiações ionizantes apresentam muitas dificuldades: são relativamente caras (equipamentos eletrônicos importados), difíceis de adquirir (fontes radioativas) e difíceis de manter (substituição das fontes de meia-vida curta). Essas restrições têm sido suficientes para dificultar a realização de experimentos deste tipo no ensino superior de física e engenharia e eliminar qualquer possibilidade de realização no ensino médio. Simulações computacionais de experimentos de física têm sido há anos utilizadas pelo seu potencial pedagógico e para mitigar estas dificuldades (DST, 2011; BIGELOW; MOLONEY; PHILPOTT; ROTHBERG, 1996; WIEMAN et al., 2011; ERNERFELDT; BODIN, 2011). Acreditamos que o experimento descrito neste artigo agrega os benefícios da simulação com poucos prejuízos advindos da falta de contato com os objetos reais. Isso se deve ao fato de que nos dois casos (o real e o simulado), o contato do experimentador com o experimento se dá em grande medida através da tela de um computador e a interface com o usuário na simulação é essencialmente a mesma oferecida pelos sistemas de aquisição de dados computadorizados. Convém reforçar, no entanto, que o real oferece benefícios inatingíveis com o virtual. O conhecimento dos riscos de manipulação de fontes radiativas, por exemplo, associados à experiência de seguir procedimentos de segurança para manipulá-las é insubstituível.

O aplicativo e as estratégias para seu uso fazem parte de um conjunto maior desenvolvido para uma disciplina de laboratório de física moderna para um curso de licenciatura em física na modalidade a distância e foi efetivamente utilizado com três turmas com cerca de 25 alunos cada em 2011 e 2014. O conjunto completo de materiais envolve um livro-texto (SILVA, 2010) que aborda quatro experimentos clássicos de física moderna. Os programas foram registrados junto ao INPI pelo Departamento de Inovação Tecnológica da UFSC, mas são de livre acesso pela Internet no endereço http://canzian.fsc.ufsc.br/labfismod/simulacoes. Outra dessas simulações, sobre atenuação da radiação pela matéria, foi extensamente explorada por Silva (2012).

$\mathrm{Na}$ Internet, é possível encontrar vários simuladores deste tipo de experimento, desde aqueles com interfaces e metodologias de cálculo sofisticadas (SAHIN; SAHIN; KAYRIN, 2011), que incorporam o estado da arte em técnicas de Monte Carlo (ALLISON et al., 2006; SALVAT; FERNÁNDEZ-VAREA; ACOSTA; SEMPAU, 2001; KAWRAKOW; MAINEGRA-HING; ROGERS; TESSIER; WALTERS, 2011), até os mais modestos (SIEGEL; SIEGEL, 2011; DIAS; PINHEIRO; BARROSO, 2002), mas que não dispõem de muita documentação mostrando as possibilidades para os menos iniciados. A proposta deste trabalho foi a de desenvolver um aplicativo de uso fácil (interface com o usuário minimalista) e rápido (sem instalação, sempre disponível na internet), com detalhados roteiros de utilização que ofereces- 
sem oportunidades de aprendizado de conceitos básicos sobre radiações ionizantes e técnicas básicas de análise e apresentação de dados. Como em um experimento real, a simulação pode ajudar a viabilizar a abordagem de temas como níveis de energia e transições atômicas e nucleares, comportamento corpuscular da luz (fótons), interação da radiação com a matéria e ciência dos materiais, aplicações de eletromagnetismo na detecção e processamento eletrônico de dados, análise estatística de dados e planejamento de experimentos.

\section{Materiais e métodos}

\section{Sistema para espectrometria gama}

Sistemas para espectrometria gama são utilizados para obter-se a distribuição em energia da radiação emitida por uma fonte (diferentemente de um detector Geiger-Müller, que apenas faz a contagem da radiação). Consistem essencialmente de três componentes: (i) um cristal cintilador, que absorve os fótons de alta energia e emite fótons de baixa energia, mais facilmente detectáveis; (ii) uma válvula fotomultiplicadora, que converte os fótons de baixa energia emitidos pelo cintilador em um fluxo intenso de elétrons proporcional à energia dos fótons incidentes; e (iii) um analisador de altura de pulso, ou analisador multicanal, que mede e registra o fluxo de elétrons proveniente do fototubo, e em geral está associado a um amplificador-formatador e integrado a um computador. A Fig. 1 ilustra, esquematicamente, estes diferentes componentes, e a Fig. 2 mostra uma fotografia de um sistema comercialmente disponível.

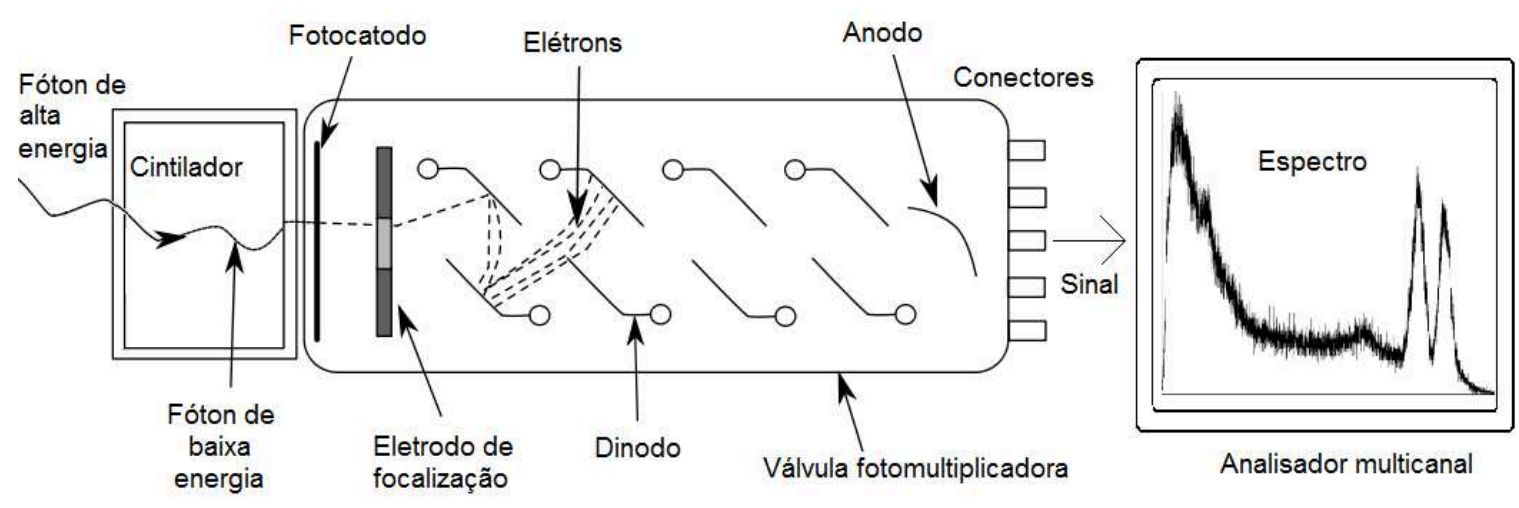

Fig. 1 - Esquema geral de um espectrômetro gama. Os fótons incidentes de alta energia (raios gama e raios $X$ da fonte radioativa) interagem com o cristal, produzindo cintilações (fótons de baixa energia, em geral visíveis e na faixa do ultravioleta), que são convertidas em fotoelétrons no fotocatodo. Estes são acelerados e multiplicados à medida que se movem entre os dinodos da base da fotomultiplicadora, em direção ao anodo, onde são coletados. O amplificador e o analisador multicanal, usualmente acoplados a um computador, registram o sinal em função da energia. 


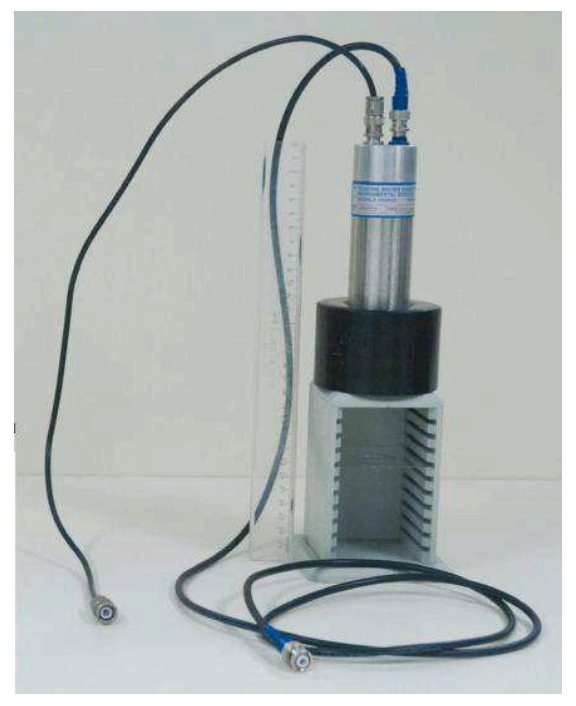

Fig. 2 - Detector de um espectrômetro de fótons. O conjunto cristal+fototubo fica dentro do invólucro de alumínio. Na parte superior encontram-se os conectores para o cabo de alta tensão e para o cabo que transmite o pulso gerado pelo evento do fototubo ao amplificador/analisador multicanal (não mostrado na imagem). O conjunto está sobre um suporte com prateleiras para posicionamento das fontes radioativas.

\section{Cristal cintilador}

O uso da cintilação produzida em certos materiais é uma das técnicas mais antigas e mais práticas para a detecção e espectroscopia da radiação. Na espectroscopia da radiação, materiais cintiladores como o $\mathrm{NaI}(\mathrm{Tl})$ (iodeto de sódio ativado com tálio) são associados a detectores de luz, tais como tubos fotomultiplicadores ou fotodiodos, que convertem o sinal luminoso em sinal elétrico.

A interação da radiação ionizante (neste caso, raios gama provenientes do decaimento do núcleo atômico ou raios $\mathrm{X}$ provenientes da reorganização da eletrosfera do átomo após o decaimento) com a matéria se dá principalmente através do efeito fotoelétrico, do espalhamento Compton e da produção de pares, com probabilidades relativas que dependem da energia do fóton incidente e do material.

No caso de efeito fotoelétrico, essencialmente toda a energia do fóton é transferida para o cristal. No caso do espalhamento Compton, eventualmente o fóton espalhado deixa o cristal sem interagir, e parte da energia do fóton incidente não é detectada. No caso da produção de pares (que só ocorre para fótons incidentes com mais de 1,02 MeV, correspondente à soma das massas de repouso do elétron e do pósitron), também parte da energia pode deixar o detector sem ser detectada, na forma de fótons produzidos na aniquilação do pósitron com um elétron do cristal.

$\mathrm{O} \mathrm{NaI}(\mathrm{Tl})$ é um cristal não condutor, o que significa que possui uma grande separação em energia entre a banda de valência, preenchida, e a banda de condução, vazia. Os elétrons produzidos pela radiação incidente irão dissipar sua energia cinética na produção de 
pares de elétrons-buracos através de colisões com os elétrons da rede cristalina. A recombinação destes pares elétron-buraco pode resultar na emissão de luz por transições radiativas ou vibrações da rede cristalina (aquecimento do cristal). A presença de tálio em uma fração molar de $10^{-3}$ aumenta significativamente a emissão de luz pelo cristal. Por causa disso o tálio é chamado ativador do cristal.

O resultado da ativação do iodeto de sódio pelo tálio é um cristal que converte aproximadamente $11 \%$ da energia dos fótons incidentes em fótons com uma energia média de 3,0 eV (máximo de emissão em $415 \mathrm{~nm}$ ). Um fóton de $1 \mathrm{MeV}$ produz, em média, 43000 fótons. A variação estatística na produção destes fótons é um dos fatores, entre vários outros, que contribuem para definir a resolução em energia.

\section{Válvula fotomultiplicadora}

Válvulas ou tubos fotomultiplicadores, ou simplesmente fototubos, são dispositivos que convertem luz em uma corrente elétrica e amplificam o sinal elétrico em várias ordens de magnitude.

O fototubo tem um catodo semitransparente na sua superfície de detecção que produz, com boa probabilidade, um elétron para cada fóton incidente (para fótons com comprimento de onda dentro do intervalo para o qual o fototubo foi projetado para ter maior eficiência). O elétron gerado no fotocatodo é acelerado através de uma sequência de eletrodos chamados dinodos, que são revestidos com um material que emite vários elétrons para cada elétron incidente, sendo o número de elétrons emitidos proporcional à energia do elétron incidente. A multiplicação eletrônica pode exceder um fator de $10^{9}$. O fator de multiplicação cresce com a diferença de potencial aplicada entre os dinodos, até um limite de saturação.

A resolução, ou capacidade de discriminação das amplitudes dos pulsos do sistema de detecção depende da coleção do maior número possível de fótons criados pela radiação incidente. $\mathrm{O}$ cristal emite fótons em todas as direções e por isso materiais altamente refletores são utilizados para revestir suas paredes externas de modo a refletir de volta o máximo possível de luz. O espaço entre o cristal e o fototubo é preenchido por graxa de silicone com índice de refração intermediário entre o do cristal e o do fototubo, de modo a minimizar reflexões a ângulos não normais à superfície. Flutuações estatísticas nestes processos também contribuem para definir a resolução em energia.

\section{Aquisição de dados}

A carga coletada no anodo é relativamente pequena. Um circuito elétrico (essencialmente um resistor e um capacitor) associado ao anodo é utilizado para coletar a carga, produzindo um pulso cuja amplitude máxima, em volts, é proporcional à carga coletada. Este pulso, em geral de dezenas a centenas de milivolts, deve ser amplificado e formatado para ficar adequado às características do sistema de aquisição de dados. A aquisição de dados é feita por um cartão especial instalado no microcomputador. Um dispositivo eletrônico no car- 
tão, o conversor analógico-digital, converte o sinal já amplificado em um número correspondente a alturas de pulso que em geral variam de 0 a 10 volts.

O software que gerencia o conjunto permite várias configurações de aquisição de dados, tais como o ajuste da diferença de potencial aplicada ao fototubo, do ganho do amplificador, do ganho de conversão do conversor analógico-digital, dos níveis inferior e superior de discriminação (eliminação de sinais abaixo e/ou acima de um valor escolhido), do tempo de aquisição, além da definição de regiões de interesse do espectro, calibração em energia, subtração da radiação de fundo, estatísticas etc.

\section{Simulação}

O programa desenvolvido simula o funcionamento de um espectrômetro de fótons associado a um analisador multicanal, isto é, um sistema que permite contar quanta radiação ionizante incide sobre o detector em função da energia. Trata-se de uma ferramenta que simula apenas os aspectos mais relevantes deste tipo de sistema, não incluindo detalhes finos sobre as emissões das fontes radioativas e sobre o processo de detecção que provavelmente não seriam relevantes no contexto a que se destina. A Fig. 3 mostra a interface do programa com o usuário, e a função dos diferentes controles é explicada na sequência.

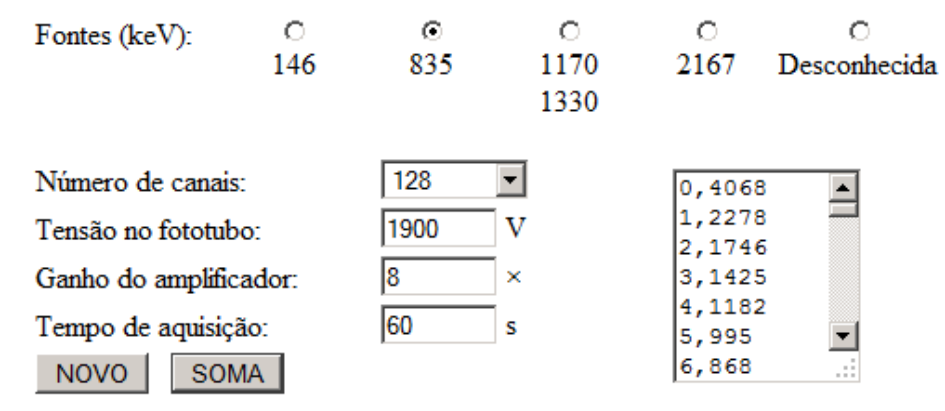

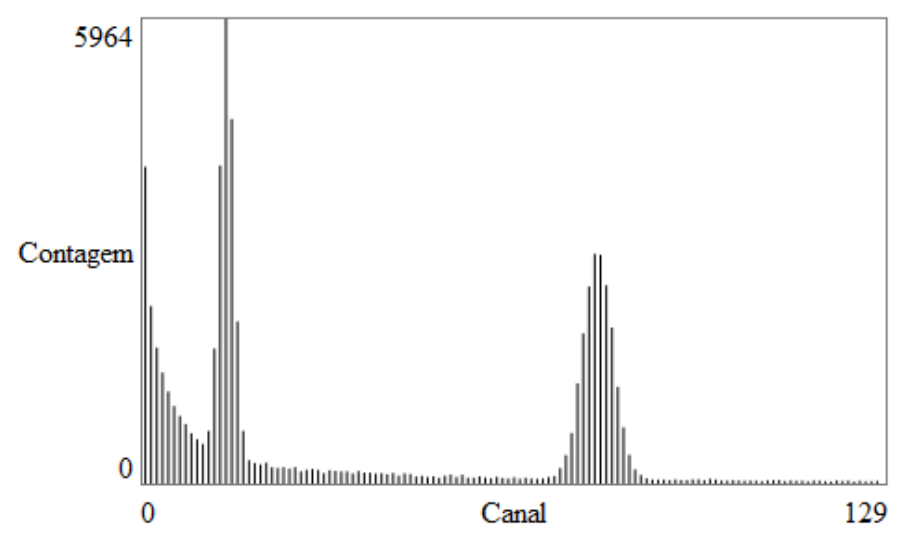

Fig. 3 - Interface da simulação do espectrômetro de fótons. Os dados simulados são mostrados na forma de um gráfico e disponibilizados como uma listagem (canal,contagem), na caixa à direita, que pode ser copiada e colada em programas de análise e visualização de dados (Origin, Excel etc.). 


\section{Fontes}

O programa simula emissões de cinco fontes, quatro delas com emissões com energias fixas (cério-141, $146 \mathrm{keV}$; manganês-54, $835 \mathrm{keV}$, cobalto-60, $1170 \mathrm{keV}$ (55\%) e 1330 $\mathrm{keV}(45 \%)$, e cloro-38, $2167 \mathrm{keV})$. A quinta fonte tem duas emissões cujas energias e intensidades são sorteadas a cada reinicialização do programa (uma das linhas tem energia entre 200 $\mathrm{keV}$ e $600 \mathrm{keV}$ e outra entre $500 \mathrm{keV}$ e $800 \mathrm{keV}$, com intensidades que variam de $20 \%$ a $80 \%$ ). As atividades de todas as fontes são sorteadas a cada reinicialização do programa, podendo variar de $500 \mathrm{~Bq}$ a $1500 \mathrm{~Bq}$.

As energias, o número de linhas e as intensidades relativas foram escolhidos de modo a cobrir relativamente bem todo o intervalo tipicamente encontrado em fontes radioativas e permitir a realização de algumas atividades didáticas que serão descritas mais adiante.

\section{Número de canais}

Também conhecido como ganho de conversão, o número de canais especifica quantas divisões acomodarão o resultado da conversão do sinal analógico de 0 a 10 volts proveniente do amplificador. A escolha do número de canais pressupõe um compromisso entre detalhamento do espectro e estatística das contagens: para um mesmo tempo de aquisição, um número de canais baixo melhora a estatística das contagens em cada canal, mas pode dificultar a percepção de detalhes do espectro. A Fig. 4 ilustra o efeito da escolha de diferentes números de canais na aquisição de um espectro.
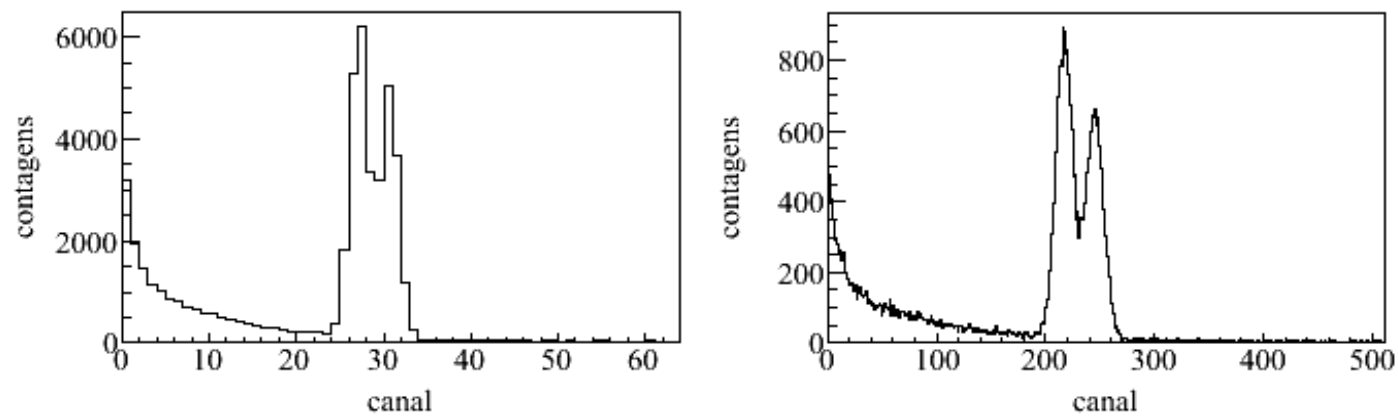

Fig. 4 - Os espectros acima foram gerados com os mesmos parâmetros, exceto pelo número de canais (ganho de conversão). À esquerda, o número de canais é 64; à direita, 512.

\section{Tensão no fototubo}

A tensão elétrica (diferença de potencial) aplicada na fotomultiplicadora define o fator de multiplicação de elétrons nos dinodos, que é tanto mais alto quanto mais alta a tensão. Se a tensão aplicada for baixa, o fator de multiplicação será baixo e a flutuação estatística no número de elétrons produzidos relativamente alta, o que piora a capacidade de resolução em energia. Tensões mais elevadas melhoram a estatística, mas podem causar danos e diminuição 
da vida útil do equipamento. A Fig. 5 ilustra o efeito de diferentes diferenças de potencial aplicadas na fotomultiplicadora.
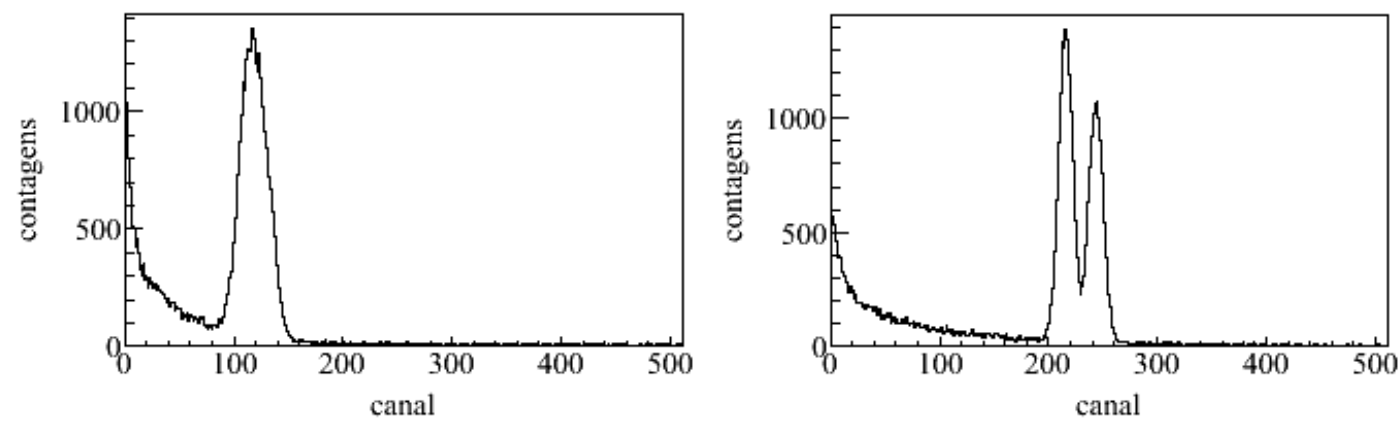

Fig. 5 - Os espectros foram gerados com os mesmos parâmetros, exceto pela tensão aplicada ao fototubo. À esquerda, a tensão é de $1200 \mathrm{~V}$; à direita, de $1800 \mathrm{~V}$. No espectro à esquerda, a flutuação estatística na multiplicação de elétrons é grande e não permite distinguir a presença das duas energias.

\section{Ganho no amplificador}

O ganho no amplificador deve ser ajustado para ajustar a altura (em volts) do pulso ao intervalo dinâmico do conversor analógico-digital, que em geral aceita pulsos de $0 \mathrm{~V}$ a 10 V. É um fator muito importante na definição de onde, no espectro, aparecerá a região de interesse. A Fig. 6 ilustra o efeito da escolha de diferentes ganhos para o amplificador.
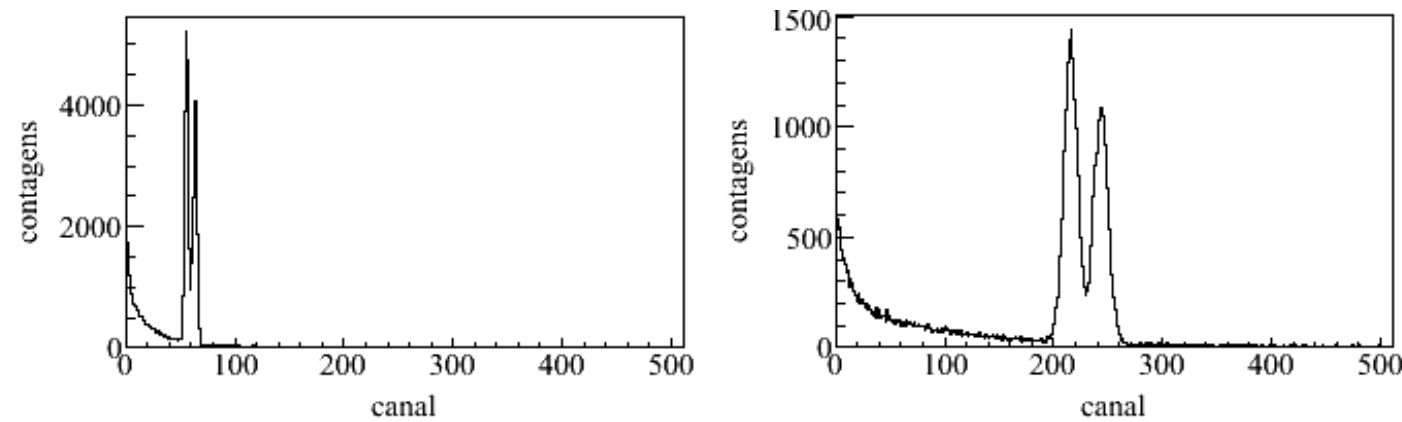

Fig. 6 - Os espectros foram gerados com os mesmos parâmetros, exceto pelo ganho do amplificador. À esquerda, o ganho é de um fator 1; à direita, de um fator 4.

\section{Tempo de contagem}

O tempo de contagem, combinado com a atividade da fonte, define o número de eventos adquiridos. Quanto maior o tempo de contagem, melhor a qualidade estatística dos dados. Esta é a grande diferença entre o experimento real e o virtual, uma vez que na simulação os resultados são gerados quase instantaneamente para qualquer tempo de aquisição escolhido. A Fig. 7 ilustra o efeito da escolha de diferentes tempos de contagem. 

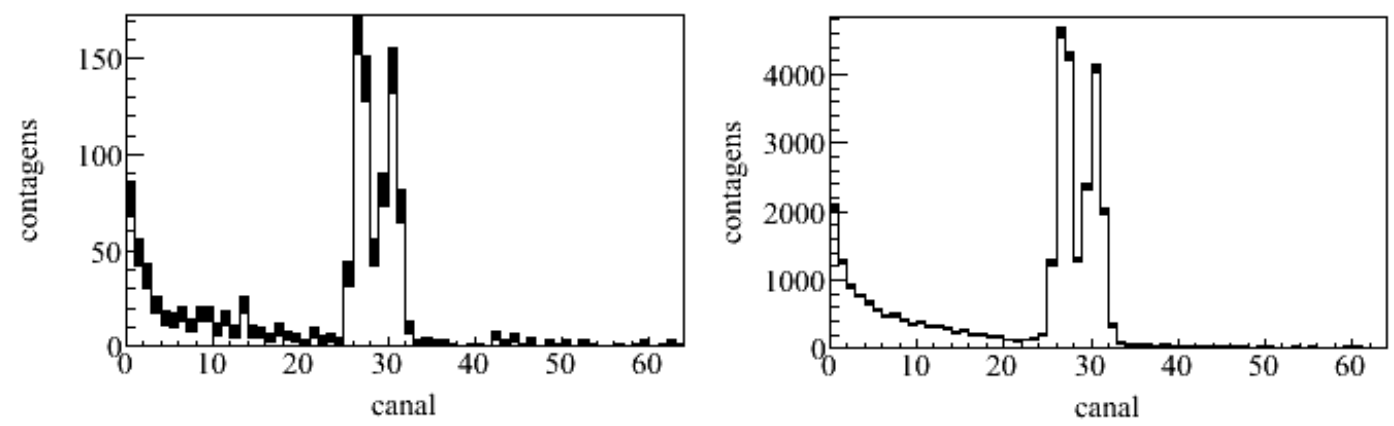

Fig. 7 - Os espectros foram gerados com os mesmos parâmetros, exceto pelo tempo de contagem. À esquerda, o tempo de contagem é de 1 segundo; à direita, de 30 segundos. As áreas escuras representam as incertezas (1 desvio padrão) na contagem registrada em cada canal. Quanto maior a contagem, menor a incerteza relativa.

\section{Cálculos}

Na simulação, o cálculo da energia efetivamente "detectada" leva em conta diversos fatores, descritos a seguir.

1. Uma pequena flutuação $( \pm 1 \%)$ no ganho geral da fotomultiplicadora, sorteado de uma distribuição uniforme a cada espectro gerado. Essa flutuação reduz-se, em última análise, à simulação de pequenas variações na diferença de potencial aplicada à fotomultiplicadora.

2. O ganho da fotomultiplicadora em função da diferença de potencial aplicada, dado por:

$$
G_{\mathrm{F}}(V)=1 /\left[1+e^{A(V-B)}\right]
$$

que descreve uma curva sigmoidal com ponto médio em $B\left(G_{\mathrm{F}}(B)=0,5\right)$ e $A$ escolhido de modo que $G_{\mathrm{F}}(V \rightarrow \infty)=1$. Na simulação foram utilizados $A=-0.007 V^{-1}$ e $B=1200 V$.

3. Uma flutuação no número total de contagens para o tempo de aquisição escolhido. Esta flutuação é calculada sorteando-se um número aleatório que obedece a uma distribuição gaussiana com média dada pelo produto da atividade da fonte pelo tempo de aquisição $(N=$ $\left.A_{\text {fte }} \times T_{\text {aq }}\right)$ e desvio padrão dado pela raiz quadrada deste valor $(\sigma=\sqrt{ } N)$, com as devidas salvaguardas para evitar a geração de números negativos.

Convém ressaltar aqui que assumimos que o número total de contagens é grande o bastante para que as flutuações estatísticas de cada componente, rigorosamente descritas por distribuições binomiais, possam ser modeladas por distribuições gaussianas que, combinadas, resultam também em uma distribuição gaussiana em que a média é dada por $N$ e cujo desvio padrão, devido ao grande valor de $N$, pode ser aproximado pela raiz quadrada desse valor, $\sqrt{ } N$.

4. Em boa parte dos eventos uma fração eventualmente significativa da energia do fóton incidente não é detectada. Num detector real são principalmente as interações fotoelétricas que contribuem para a formação do 'pico' no espectro (fotopico). As interações por espalhamento Compton levam em geral a uma não detecção de parte da energia do fóton inciden- 
te, gerando um espectro de fundo cuja forma depende essencialmente da geometria do detector (não simulado). Nesta simulação, assumiu-se que, do total de contagens, somente $50 \%$ estariam sob o fotopico.

5. Uma pequena não linearidade no ganho geral (fotomultiplicadora + amplificador + conversor analógico-digital). Seria desejável que o sistema de aquisição incrementasse as contagens nos canais $C$ da distribuição em função da energia $E$ de tal modo que $C=a E$, onde $a$ é uma constante de proporcionalidade. A realidade, entretanto, é mais complexa, e para chamar a atenção para isto foi introduzida uma não-linearidade quadrática, de modo que $C=$ $a\left(E+10^{-5} E^{2}\right)$.

6. Um espectro de fundo genérico, sem levar em conta qualquer hipótese a respeito da sua real composição (a borda Compton, por exemplo), foi simulado utilizando três componentes exponenciais dadas por:

$$
h(E)=A_{1} \mathrm{e}^{-\lambda} 1^{E}+A_{2} \mathrm{e}^{-\lambda} 2^{E}+A_{3} \mathrm{e}^{-\lambda} 3^{E}
$$

onde $\lambda_{1}=0,1 / E_{\text {máx }}$ para a componente 'rápida', $\lambda_{2}=3 / E_{\text {máx }}$ para a componente 'intermediária' e $\lambda_{3}=30 / E_{\text {máx }}$ para a componente 'lenta', $E_{\text {máx }}$ corresponde ao valor nominal da energia das emissões mais energéticas da fonte escolhida, e os valores de $A_{1}, A_{2}$ e $A_{3}$ foram escolhidos de modo que suas contribuições na contagem total do fundo fossem de $40 \%$, $50 \%$ e $10 \%$, respectivamente.

Como visto, foram utilizados geradores de números aleatórios que obedecem a distribuições exponenciais e gaussianas. Funções que geram números assim não são parte das linguagens básicas de programação, mas podem ser construídos a partir de funções que geram números aleatórios que obedecem distribuições uniformes, em geral disponíveis.

Um gerador de números aleatórios y que obedecem a uma distribuição exponencial $p(y) d y=\lambda e^{-\lambda y} d y$ pode ser construído a partir de um gerador de números aleatórios $x$ que obedecem a uma distribuição uniforme $p(x) d x=d x$ segundo a relação (PRESS et. al., 2002):

$$
y=-(1 / \lambda) \ln (x)
$$

Um gerador de números aleatórios y que obedecem a uma distribuição gaussiana $p(y) d y=(1 / 2 \pi)^{1 / 2} \mathrm{e}^{-y 2 / 2} d y$ pode ser construído a partir de um gerador de números aleatórios $x$ que obedecem a uma distribuição uniforme $p(x) d x=d x$ utilizando o método de Box-Muller (PRESS et. al., 2002). Com este método é possível gerar, a cada ciclo, dois números $y_{1}$ e $y_{2}$ a partir de dois números $x_{1}$ e $x_{2}$ segundo as equações:

$$
y_{1}=-2 \ln \left(x_{1}\right) \cos \left(2 \pi x_{2}\right) \quad \text { e } \quad y_{2}=-2 \ln \left(x_{1}\right) \operatorname{sen}\left(2 \pi x_{2}\right)
$$

A simulação foi desenvolvida em JavaScript (ECMA, 2010), uma linguagem que, no nosso entendimento, oferece vantagens para este tipo de aplicação não encontradas em qualquer outra plataforma de desenvolvimento. 


\section{Atividades propostas}

A seguir, são apresentadas duas atividades em geral realizadas com este tipo de equipamento no laboratório real e que podem ser reproduzidas com razoável fidelidade com o uso da simulação.

\section{Curva característica de um espectrômetro}

A resposta de um espectrômetro composto de um cristal de $\mathrm{NaI}(\mathrm{Tl})$ e um fototubo depende crucialmente da diferença de potencial elétrico aplicada entre os dinodos do fototubo. Diferenças de potencial baixas correspondem a baixos fatores de amplificação e menor resolução. Diferenças de potencial elevadas demais podem danificar irremediavelmente o equipamento ou diminuir a sua vida útil.

Para o funcionamento otimizado, é necessário que um elétron acelerado entre um dinodo e outro atinja uma velocidade tal que, no impacto com o dinodo-alvo, provoque a emissão do maior número possível de elétrons secundários. A resolução do sistema também melhora paulatinamente com o aumento da diferença de potencial aplicada: quanto maior, melhor a coleção de cargas e menor o ruído eletrônico, que é proporcional à raiz quadrada do número de elétrons coletados.

A quantidade de elétrons emitidos é função crescente da diferença de potencial: quanto maior for, maior a energia do elétron ao atingir o dinodo e maior o número de elétrons secundários emitidos. Entretanto, a partir de certo valor de tensão ocorre a saturação e o aumento da diferença de potencial acima do valor de saturação não mais provoca um aumento na emissão de elétrons. Ao contrário, valores muito elevados podem danificar o equipamento. A escolha do ponto ideal pode ser feita a partir de algumas curvas características do detector. Curvas características são gráficos dos parâmetros relevantes - posição de um pico de referência, largura a meia altura, resolução fracional - em função da diferença de potencial aplicada ao fototubo, a partir dos quais pode-se inferir a tensão de saturação.

A qualidade de um sistema de detecção está intimamente associada à largura de um pico relativamente à sua posição no espectro. A resolução fracional $\Delta E / E$ do sistema, uma medida desta qualidade, é usualmente definida como a razão da largura a meia altura (FWHM, do inglês full width at half maximum) do pico dividida pelo valor da posição da centroide. A partir de argumentos puramente estatísticos, pode-se mostrar que, para uma distribuição gaussiana, a largura a meia altura corresponde a 2,35 vezes o desvio padrão da média $(\mathrm{FWHM}=2,35 \sigma)$ e que a resolução melhora com o inverso da raiz quadrada da energia:

$$
\Delta E / E \quad \propto \quad E^{1 / 2} / E=1 / E^{1 / 2}
$$

A resolução fracional essencialmente nos revela qual a capacidade do sistema de "resolver", ou separar, duas estruturas distintas. Por exemplo, para um pico centrado em 1000 $\mathrm{keV}$ uma resolução fracional de $10 \%$ representa $100 \mathrm{keV}$. Isto significa que fica muito difícil dizer se o pico representa as contagens de apenas uma linha de emissão da fonte ou se este 
pico é a soma de dois ou mais picos separados em energia por menos de $100 \mathrm{keV}$ (na verdade, existem técnicas de análise da forma do pico que permitem ir um pouco mais longe neste aspecto).

Os gráficos da Fig. 8 ilustram a questão. Os três gráficos foram obtidos exatamente nas mesmas condições (fonte com fótons de $835 \mathrm{keV}, 1024$ canais, ganho de $8 \times$ no amplificador e $60 \mathrm{~s}$ de tempo de aquisição), exceto pela diferença de potencial aplicada ao fototubo.
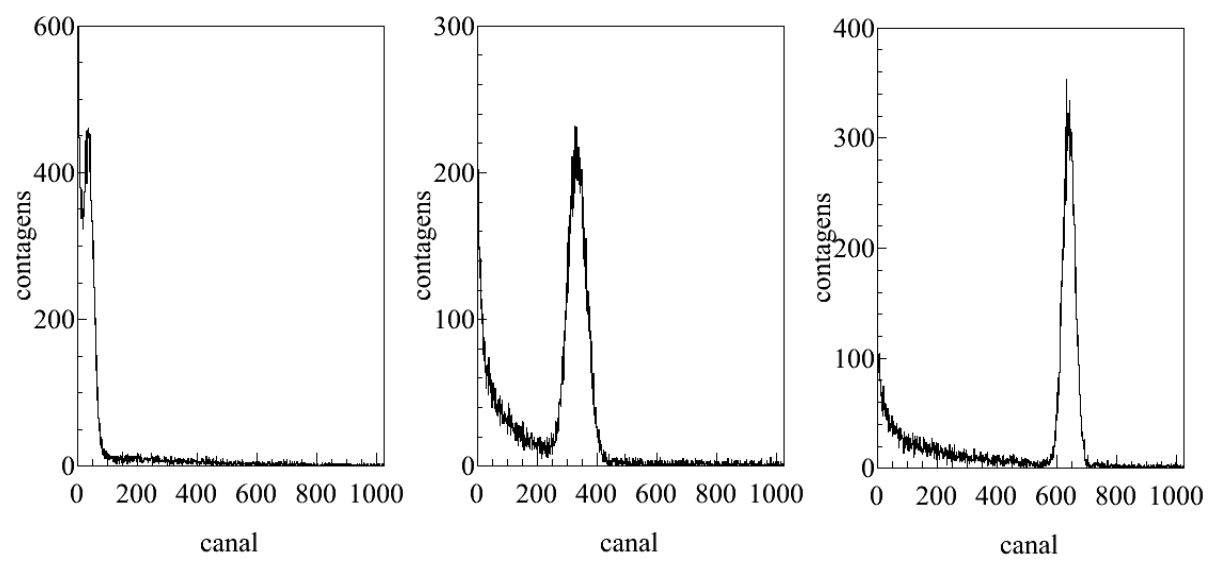

Fig. 8 - Simulação da aquisição de dados com um espectrômetro de NaI(Tl) utilizando uma fonte com emissões gama de $835 \mathrm{keV}$. As condições de aquisição foram as mesmas nos três casos (fonte com fótons de $835 \mathrm{keV}, 1024$ canais, ganho de $8 \times$ no amplificador e 60 $s$ de tempo de aquisição), exceto pela diferença de potencial, de $800 \mathrm{~V}$ (esquerda), $1200 \mathrm{~V}$ (centro) e $2000 \mathrm{~V}$ em (direita).

A posição do máximo varia muito com a diferença de potencial aplicada: em torno do canal 40 para $800 \mathrm{~V}$, do canal 360 para $1200 \mathrm{~V}$ e do canal 720 para $2000 \mathrm{~V}$. Isso significa que aumentando a diferença de potencial de $800 \mathrm{~V}$ para $1200 \mathrm{~V}$, o número de elétrons coletados para cada fóton incidente quase decuplica e aumentando de $1200 \mathrm{~V}$ para $2000 \mathrm{~V}$ o número de elétrons coletados dobra.

A resolução também melhora substancialmente. No primeiro caso, o pico de 835 keV mistura-se com a radiação de fundo, e apesar de não fazer muito sentido, podemos estimar a sua largura a meia altura em cerca de 50 canais, o que corresponde a uma resolução fracional de $50 / 40 \times 100 \%=125 \%$. No segundo caso a largura a meia altura é de cerca de 90 canais, o que para um pico próximo ao canal 300 corresponde a uma resolução de 90/300 × $100 \%=30 \%$. No terceiro caso, a largura a meia altura é de 60 canais, e o pico da distribuição está próximo ao canal 600, o que corresponde a uma resolução de cerca de $10 \%$.

A tabela 1 mostra as posições dos picos e larguras à meia altura das distribuições em função da tensão aplicada no fototubo. Os dados foram simulados para uma fonte de $835 \mathrm{keV}$, ganho de conversão de 1024 canais, ganho de 8 no amplificador e tempo de aquisição de 60 segundos. A Fig. 9 apresenta gráficos construídos com estes dados. 
Tabela 1 - Posição do pico de $835 \mathrm{keV}$ em função da diferença de potencial aplicada ao fototubo. A posição do pico reflete o aumento do fator de multiplicação do fototubo. Note a saturação em torno dos $1800 \mathrm{~V}$.

\begin{tabular}{|ccc|ccc|}
\hline $\begin{array}{c}\text { Tensão no } \\
\text { fototubo } \\
\text { (V) }\end{array}$ & $\begin{array}{c}\text { Posição } \\
\text { do pico } \\
\text { (canal) }\end{array}$ & $\begin{array}{c}\text { Larg. } \\
\text { a meia } \\
\text { altura } \\
\text { (canais) }\end{array}$ & $\begin{array}{c}\text { Tensão no } \\
\text { fototubo } \\
\text { (V) }\end{array}$ & $\begin{array}{c}\text { Posição } \\
\text { do pico } \\
\text { (canal) }\end{array}$ & $\begin{array}{c}\text { Larg. } \\
\text { a meia } \\
\text { altura } \\
\text { (canais) }\end{array}$ \\
\hline 800 & 38 & 49 & 1600 & 686 & 66 \\
900 & 76 & 63 & 1700 & 707 & 63 \\
1000 & 142 & 78 & 1800 & 717 & 61 \\
1100 & 240 & 87 & 1900 & 724 & 61 \\
1200 & 364 & 92 & 2000 & 726 & 61 \\
1300 & 486 & 85 & 2250 & 728 & 61 \\
1400 & 584 & 78 & 2500 & 728 & 61 \\
1500 & 649 & 71 & 2750 & 728 & 61 \\
\hline
\end{tabular}
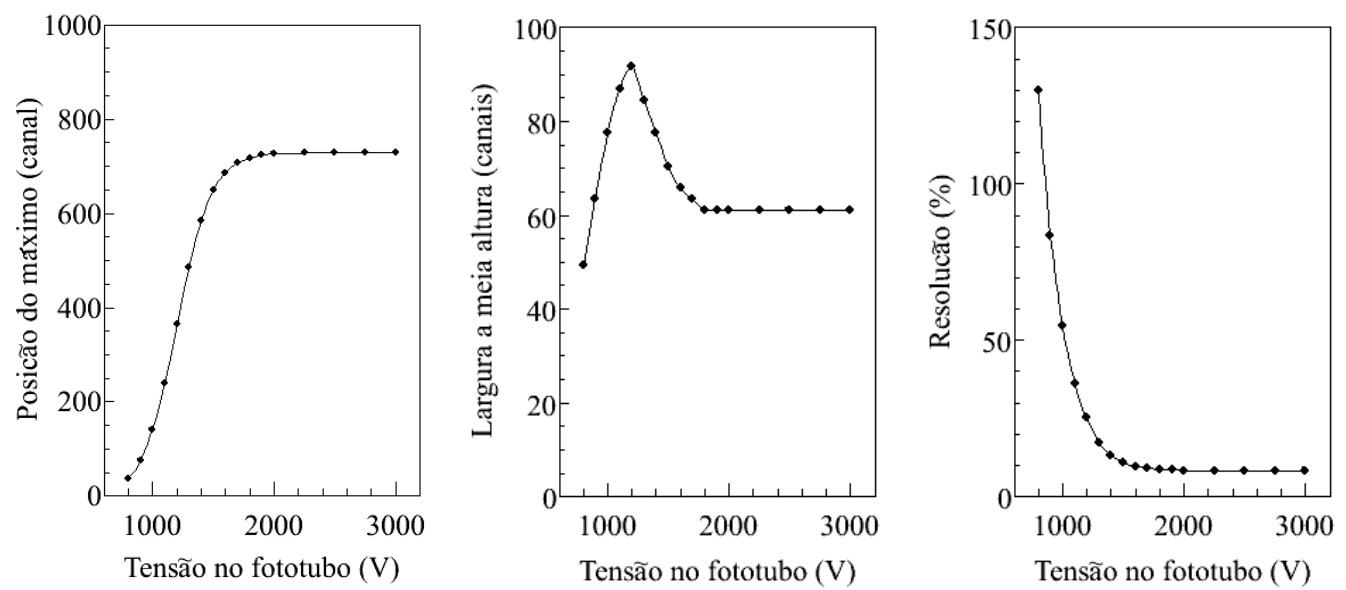

Fig. 9 - Posição (esquerda), largura a meia altura (centro) e resolução (direita) para o pico de $835 \mathrm{keV}$ em função da diferença de potencial no fototubo. Em todos os casos, nota-se a saturação em torno dos $1800 \mathrm{~V}$.

\section{Calibração em energia}

A Fig. 10 mostra um espectro real obtido com uma fonte de ${ }^{22} \mathrm{Na}$ com o espectrômetro gama do Laboratório de Física Moderna da UFSC. O espectro do ${ }^{22} \mathrm{Na}$ tem três picos de energia bem definida: $511 \mathrm{keV}, 1275 \mathrm{keV}$ e $1786 \mathrm{keV}$, centrados nos canais 370, 668 e 816 respectivamente. O pico de $1786 \mathrm{keV}$ é muito pequeno, possível de ser visto claramente somente ampliando-se a figura ou utilizando uma escala logarítmica para o eixo vertical. $\mathrm{O}$ espectro contém ainda um pequeno pico em torno do canal 70, correspondente a raios $\mathrm{X}$ de baixa energia, e uma estrutura de fundo complexa, devida principalmente à coleção incompleta 
da energia dos fótons secundários do espalhamento Compton. As energias e posições dos picos no espectro podem ser utilizadas para calcular a transformação que leva o número de qualquer canal para um valor de energia.

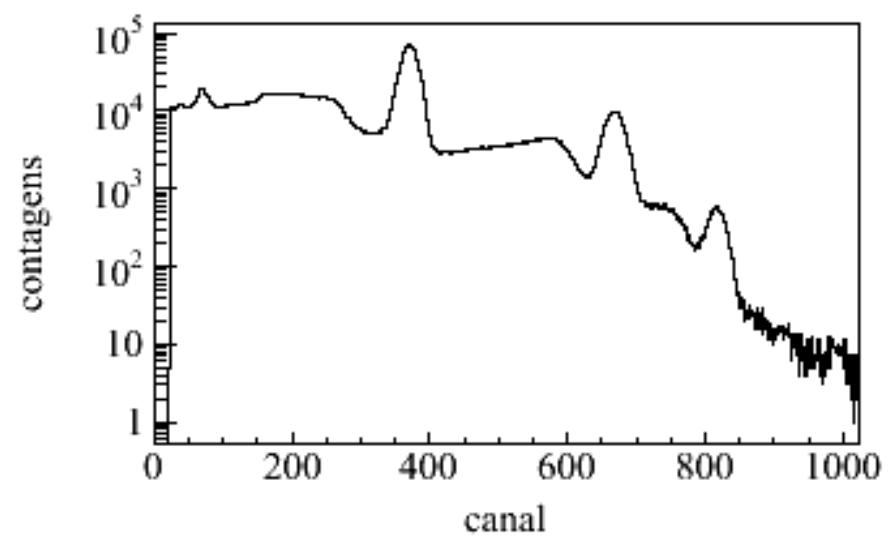

Fig. 10 - Espectro de uma fonte de ${ }^{22} \mathrm{Na}$ obtido com um espectrômetro de fótons. Os picos, centrados nos canais 370, 668 e 816, correspondem a energias de $511 \mathrm{keV}, 1275 \mathrm{keV}$ e $1786 \mathrm{keV}$. Os dados são apresentados em uma escala vertical logarítmica para que seja possível ver o pico de $1786 \mathrm{keV}$.

O problema é que em geral o sistema de aquisição não é linear. Note que entre o pico de $511 \mathrm{keV}$ e o de $1275 \mathrm{keV}$, intervalo que corresponde a $764 \mathrm{keV}$, existem 298 canais, de modo que 1 canal = 2,56 keV, em média. Já no intervalo entre $1275 \mathrm{keV}$ e $1786 \mathrm{keV}, 1$ canal $=3,45 \mathrm{keV}$, em média. Idealmente, para uma adequada calibração de todo o espectro, utilizam-se polinômios de segundo grau, desde que se tenha pelo menos três picos com energias conhecidas. Para fins didáticos, entretanto, um simples ajuste linear em cada região de interesse, que pode ser feito com uma calculadora de mão ou com aplicativos abundantemente disponíveis na internet, já serve para sensibilizar os estudantes para o problema.

No caso dos espectros acima, a calibração dos intervalos seria a descrita na tabela abaixo. Desse modo, quando se deseja saber a energia de pontos entre os picos de $511 \mathrm{keV}$ e de $1275 \mathrm{keV}$, utiliza-se uma calibração, e sobre pontos entre os picos de $1275 \mathrm{keV}$ e 1786 $\mathrm{keV}$, utiliza-se a outra. A tabela 2 mostra os parâmetros da calibração em energia do espectro de ${ }^{22} \mathrm{Na}$ para as duas regiões entre os picos.

Tabela 2 - Calibração em energia utilizando um ajuste linear para cada região de interesse do espectro de ${ }^{22} \mathrm{Na}$ da Fig. 10.

\begin{tabular}{|c|c|}
\hline 511 keV a 1275 keV & 1275 keV a 1786 keV \\
\hline $511=a+b \cdot 370$ & $1275=a+b \cdot 668$ \\
$1275=a+b \cdot 668$ & $1786=a+b \cdot 816$ \\
$a=-437 \mathrm{keV}$ & $a=-1030 \mathrm{keV}$ \\
$b=2,56 \mathrm{keV} /$ canal & $b=3,45 \mathrm{keV} / \mathrm{canal}$ \\
$E=-437+2,56 \cdot C$ & $E=-1030+3,45 \cdot C$ \\
\hline
\end{tabular}


A mesma metodologia pode ser aplicada com dados obtidos com a simulação. A Fig. 11 mostra um espectro composto de quatro 'aquisições' de $60 \mathrm{~s}$, todas utilizando $1800 \mathrm{~V}$ para a diferença de potencial aplicada ao fototubo, ganho de 5 vezes no amplificador e ganho de conversão de 1024 canais. Três delas foram feitas com fontes conhecidas e uma com a fonte desconhecida. Os picos com energias conhecidas são os correspondentes a $146 \mathrm{keV}, 1179$ $\mathrm{keV}, 1330 \mathrm{keV}$ e $2167 \mathrm{keV}$, centrados nos canais 73, 556, 628 e 982, respectivamente. Os dois picos com energias desconhecidas estão centrados nos canais 194 e 347 . A tabela 3 mostra os resultados para os ajustes lineares nos dois segmentos.

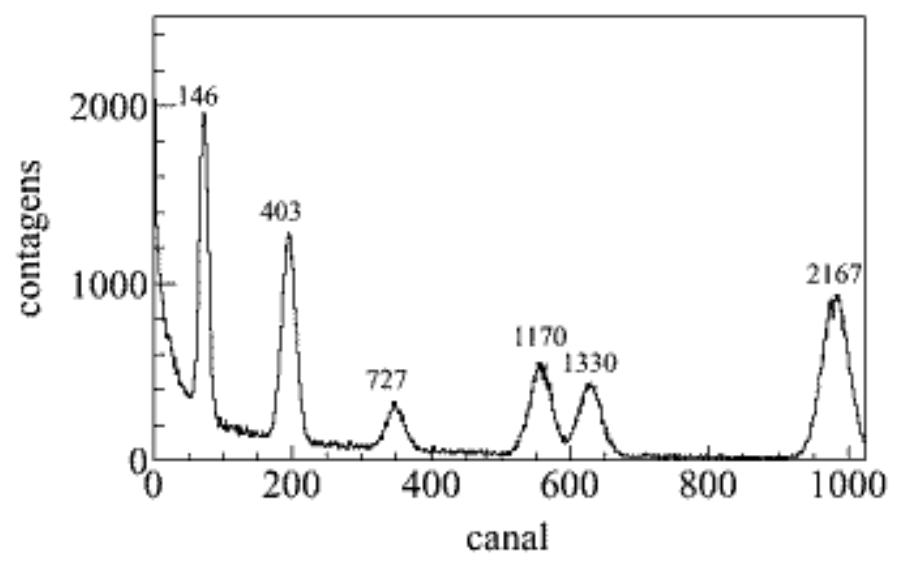

Fig. 11 - Espectro simulado, composto de 4 aquisições de 60 s para 3 fontes com emissões com energias conhecidas (146 keV, $1179 \mathrm{keV}, 1330 \mathrm{keV}$ e $2167 \mathrm{keV})$ e uma com duas com energias desconhecidas, determinadas após a calibração como correspondentes a $403 \mathrm{keV} \mathrm{e} 727 \mathrm{keV}$.

Tabela 3 - Calibração em energia utilizando um ajuste linear para cada região de interesse do espectro composto apresentado na Fig. 11.

\begin{tabular}{|c|c|}
\hline $146 \mathbf{~ k e V ~ a ~ 1 1 7 0 ~ k e V ~}$ & 1330 keV a 2167 keV \\
\hline $146=a+b \cdot 73$ & $1330=a+b \cdot 628$ \\
$1170=a+b \cdot 556$ & $2167=a+b \cdot 982$ \\
$a=-8,72 \mathrm{keV}$ & $a=-154,84 \mathrm{keV}$ \\
$b=2,12 \mathrm{keV} / \mathrm{canal}$ & $b=2,36 \mathrm{keV} / \mathrm{canal}$ \\
$E=-8,72+2,12 \cdot C$ & $E=-154,84+2,36 \cdot C$ \\
\hline
\end{tabular}

\section{Reflexões sobre a proposta}

Como fizemos no artigo sobre outra das simulações do conjunto (SILVA, 2012), podemos tentar analisar como este trabalho se relaciona às principais metas dos laboratórios introdutórios de física, segundo apontadas por um documento (TAYLOR et al., 1998) produzido por um comitê da American Physical Society estabelecido para esse fim. As principais metas dos laboratórios introdutórios de física seriam fazer com que os estudantes desenvol- 
vam: 1) a arte da experimentação; 2) habilidades de experimentação e análise; 3) aprendizagem conceitual; 4) entendimento do conhecimento de base da física; 5) habilidades de aprendizado colaborativo.

\section{Arte da experimentação}

As atividades laboratoriais devem "engajar os estudantes em vivências significativas do processo experimental, incluindo alguma experiência em propor investigações" e auxiliar os estudantes a compreender que "a física é uma ciência experimental e que a observação e a experimentação são tão importantes quanto conceitos e teorias".

Entendemos que a simulação apresentada cumpre parte desse objetivo. Obviamente não permite um aprimoramento das habilidades visuais e manipulativas finas que fazem um bom experimentador, tais como posicionamento de fontes, organização do laboratório e da bancada, ligações de cabos, ajuste de potenciômetros, e inúmeras outras minúcias que são tidas frequentemente como meros detalhes técnicos, mas que promovem reflexões sobre o processo e, em mentes bem preparadas, visões sobre outras aplicações e melhoramentos.

Ainda assim, a simulação permite a manipulação e reflexões sobre variáveis que surgem exclusivamente do processo experimental: detalhes da estrutura nuclear não têm nada a ver com tensões no fototubo, ganhos no amplificador ou analisadores multicanais, mas estes são essenciais para a sua compreensão. Além disso, são instrumentos e metodologias amplamente utilizadas em outros contextos, tais como nas aplicações da física na medicina e na caracterização de materiais.

\section{Habilidades de experimentação e análise}

Os autores argumentam que "o laboratório deve ajudar o estudante a desenvolver uma vasta gama de habilidades básicas e de uso de ferramentas de física experimental e análise de dados" e que "é recomendável fazer com que os estudantes utilizem muitos tipos de equipamentos básicos de laboratório para fazer observações".

Nesse quesito, a simulação, como qualquer outra que não use maquetes de equipamentos, deixa a desejar. Experimentos virtuais não contemplam alguns itens tais como o cuidado necessário ao manipular equipamentos, a atenção na realização de conexões de cabos de energia e de sinais, a manutenção da integridade dos equipamentos, o alinhamento entre as peças e sensibilidade para as consequências da degradação natural dos equipamentos. Entretanto, no que se refere à análise da informação, a simulação oferece visualização básica e mecanismos de exportação de dados semelhantes aos equipamentos reais e que podem ser amplamente explorados pelas ferramentas de escolha do usuário.

Uma diferença fundamental entre o real e o virtual é a passagem do tempo (proposital, pois também poderia ter sido simulada): experimentos que poderiam levar horas no laboratório real são executados em poucos minutos no laboratório virtual. Se por um lado isso permite explorar aspectos que não seriam possíveis de serem explorados no laboratório real 
nas condições didáticas, por outro lado oferece uma perspectiva deformada desse aspecto do processo experimental.

Por fim, os autores argumentam que "o computador é uma importante ferramenta para um curso de física baseado na investigação porque tornou-se a mais universal das ferramentas de investigação na pesquisa científica", e neste sentido o experimento virtual tem uma contribuição em tudo semelhante à do experimento real.

\section{Aprendizagem conceitual}

Aqui entendemos a referência à aprendizagem conceitual como relacionada aos fundamentos das técnicas, dos equipamentos e das metodologias de análise de dados (o conhecimento de base em física será explorado no próximo item). Nesse sentido, entendemos que o virtual se aproxima muito do real, pelo menos no caso das abordagens mais básicas. A simulação implementa muitos dos elementos fundamentais sobre a física e a estatística da emissão e detecção das radiações ionizantes. Para citar uma diferença, podemos dizer que um laboratório real possui uma gama maior de equipamentos (multímetro e osciloscópio, por exemplo) que podem ser empregados para incrementar o aprendizado sobre o processo de aquisição de dados e que são itens importantes para formação de um bom físico experimental.

\section{Entendimento do conhecimento de base}

Nesse aspecto, entendemos que a realização roteirizada do experimento, seja real ou virtual, dificilmente contribui para o aprimoramento do conhecimento de base. Esse não é o caso, entretanto, quando há abundante material de apoio e a metodologia e do professor contribuem para uma atitude investigativa por parte dos estudantes. No caso dessa simulação, o livro-texto associado contempla, ainda que superficialmente, noções sobre a estrutura nuclear que dá origem aos espectros (não explorados nesse artigo) e informações técnicas a respeito do funcionamento interno do sistema de detecção (parcialmente descritos nesse artigo).

\section{Habilidades de aprendizado colaborativo}

No que se refere ao desenvolvimento de habilidades para a aprendizagem colaborativa, o experimento virtual tende a ser mais solitário devido à interação exclusiva com o computador. Não existem peças a serem manipuladas, e mesmo se a experiência for realizada em equipe, em geral apenas um dos integrantes tem uma intensa interação com o teclado e o mouse. Por outro lado, a própria proposta de realizar simulações deste tipo nasce de uma demanda específica, a de atingir pessoas que não têm oportunidade ou meios de participar intensamente de um laboratório real e colaborativo. 


\section{Conclusão}

A simulação apresentada nesse trabalho tem sido utilizada, no nosso entendimento, com um elevado grau de aproveitamento por parte dos alunos de todas as edições da disciplina de Laboratório de Física Moderna do curso de Licenciatura em Física a distância da UFSC e eventualmente pelos alunos do curso presencial. No curso a distância, em que toda a disciplina é concentrada em dois fins de semana consecutivos, entendemos que o seu uso tem sido particularmente significativo. Antes da fase presencial, através de exercícios e atividades prédefinidas, é utilizada para sensibilizar e preparar os alunos; após a fase presencial, durante a elaboração dos relatórios, serve para ajudá-los a dirimir dúvidas e explorar um pouco mais os experimentos. Esperamos compreender melhor o papel dessas simulações através de uma pesquisa que foi realizada durante a edição de 2014 e que está em vias de tornar-se uma dissertação de mestrado a ser defendida em meados de 2015.

\section{Referências}

ALLISON, J. et al. Geant4 Developments and Applications. IEEE Transactions on Nuclear Science, v. 53, n. 1, February 2006.

ATLAS Collaboration et al. The ATLAS Experiment at the CERN Large Hadron Collider. Journal of Instrumentation, v. 3, August 2008. DOI: 10.1088/1748-0221/3/08/S08003.

BIGElOW, R.; MOLONEY, M. J.; PHILPOTT, J.; ROTHBERG, J. Nuclear and Particle Physics Simulations: The Consortium of Upper-Level Physics Software. WILEY, 1996.

BRENNER, D. J. Are X-Ray Backscatter Scanners Safe for Airport Passenger Screening? For Most Individuals, Probably Yes, but a Billion Scans per Year Raises LongTerm Public Health Concerns. Radiology, v. 259, p. 6-10, April 2011.

DIAS, N. L.; PINHEIRO, A. G.; BARROSO, G. C. Laboratório Virtual de Física Nuclear. Revista Brasileira de Ensino de Física, v. 24, n. 2, p. 232-236, Jun. 2002.

DST (Design Simulation Technologies, Inc.) Interactive Physics. Disponível em: <http://www.design-simulation.com/IP/>. Acesso em 23 set. 2014.

ECMA International. ECMAScript-262 Language Specification, 5th. Edition. Ecma International, 2009. Disponível em: <http://www.ecma-international.org/publications/files/ECMAST/ECMA-262.pdf>. Acesso em: 22 set. 2014.

ERNERFELDT, E.; BODIN, K. PHUN! Disponível em: <http://phun.en.softonic.com/>. Acesso em: 23 set. 2014. 
FAIRAND, B. P.; FIDOPIASTIS, N. Radiation Sterilization of Aseptically Manufactured Products. PDA Journal of Pharmaceutical Science and Technology, v. 64, n. 4, p. 299-304, July/August 2010.

GIBLIN, P.; LIPTON, E. New Airport X-Rays Scan Bodies, Not Just Bags. The New York Times, February 24, 2007. Disponível em:

<http://www.nytimes.com/2007/02/24/us/24scan.html>. Acesso em 23 set. 2014.

GUIDOLIN, R.; CORREAA, A.; CICARELLI, R. M. B.; PREVIDE, E.; MORAIS, J. F.; HIGASHI, H. G. Esterilização de soros e vacinas por radiação gama de cobalto. Revista de Saúde Pública, São Paulo, v. 22, n. 2, p. 113-7, 1988.

GÜDEL, M. A decade of X-ray astronomy with XMM-Newton. Astronomy and Astrophysics, v. 500, n. 1, p. 595-596, June II 2009. DOI 10.1051/0004-6361/200912208.

IPEN (Instituto de Pesquisas Energéticas e Nucleares), Jornal Órbita, de 01/05/2003. Irradiação garante esterilização de materiais médicos e cirúrgicos. Disponível em:

<http://www.ipen.br/sitio/?idc=814>. Acesso em: 23 set. 2014.

JACOBS, G. P. A Review of the Effects of Gamma Radiation on Pharmaceutical Materials. Journal of Biomaterials Applications, v. 10, n. 1, p. 59-96, July 1995.

KAWRAKOW, I.; MAINEGRA-HING, E.; ROGERS, D. W. O.; TESSIER, F.; WALTERS, B. R. B. The EGSnrc Code System: Monte Carlo Simulation of Electron and Photon Transport. National Research Council of Canada Report PIRS-701, May 9, 2011.

MAITY, J. P.; KARA, S.; BANERJEE, S.; CHAKRABORTY, A.; SANTRA, S.C. Effects of gamma irradiation on long-storage seeds of Oryza sativa (cv. 2233) and their surface infecting fungal diversity. Radiation Physics and Chemistry, v. 78, n. 11, p. 1006-1010, November 2009.

PRESS, W. H.; TEUKOLSKY, S. A.; VETTERLING, W. T.; FLANNERY, B. P. Numerical Recipes in C: The Art of Scientific Computing, Second Edition. Cambridge: Cambridge University Press, 2002.

SAHIN, D.; SAHIN, S.; KAYRIN, K. RADLab - Virtual radiation detection experiment open source software. Disponível em: <http://radlab.sourceforge.net>. Acesso em: 23 set. 2014.

SAlVAT, F.; FERnÁNDEZ-VAREA, J. M.; ACOSTA, E.; SEMPAU, J. PENELOPE: A Code System for Monte Carlo Simulation of Electron and Photon Transport. Nuclear Energy Agency, Organisation for Economic Co-operation and Development, 2001.

SIEGEL, P.; SIEGEL, J. California State Polytechnic University Physics Department's Virtual Radiation Laboratory. Disponível em: 
<http://www.csupomona.edu/ pbsiegel/vertuallab/vertlab.html>. Acesso em: 23 set. 2014.

SILVA, N. C. Laboratório de Física Moderna. Universidade Federal de Santa Catarina, Florianópolis, 2010.

SILVA, N. C. Laboratório virtual de física moderna: atenuação da radiação pela matéria. Caderno Brasileiro de Ensino de Física, v. 29, n. 3, p. 1206-1231, dezembro de 2012. DOI 10.5007/2175-7941.2012v29n3p1206.

STOCK, S. R. Recent advances in X-ray microtomography applied to materials. International Materials Reviews, v. 53, n. 3, p. 129-181, May 2008.

TAYLOR, G. (Org.). Goals of the introductory Physics laboratory. American Journal of Physics, v. 66, n. 6, June 1998.

VALENCIA, J.; MILLER, S. X-ray scan detection for cargo integrity Proc. SPIE 7983, 79830X, 2011. Doi:10.1117/12.882003. In: CONFERENCE: NONDESTRUCTIVE CHARACTERIZATION FOR COMPOSITE MATERIALS, AEROSPACE ENGINEERING, CIVIL INFRASTRUCTURE, AND HOMELAND SECURITY, 2011, San Diego, California, USA.

WATSON, J. D.; CRICK, F. H. C. Molecular structure of nucleic acids: a structure for the deoxyribose nucleic acid. Nature, v. 171, p. 737-738, April 1953.

WIEMAN, C. et al. PHET. Disponível em: <http://phet.colorado.edu/>. Acesso em: 23 set. 2014. 